\title{
Laparoscopic Surgery and Robot-Assisted Surgery for Colorectal Cancer With Persistent Descending Mesocolon
}

\author{
Yutaka Kojima ( $\nabla$ yutachan@juntendo.ac.jp ) \\ Juntendo University Faculty of Medicine https://orcid.org/0000-0001-7825-1828
}

Kazuhiro Sakamoto

Juntendo University Faculty of Medicine

Masaya Kawai

Juntendo University Faculty of Medicine

Yu Okazawa

Juntendo University Faculty of Medicine

Kazumasa Kure

Juntendo University Faculty of Medicine

Aya Kobari

Juntendo University Faculty of Medicine

\section{Case report}

Keywords: Laparoscopic surgery, Robot surgery, Persistent descending mesocolon, Colorectal cancer

Posted Date: August 24th, 2020

DOI: https://doi.org/10.21203/rs.3.rs-63249/v1

License: () (1) This work is licensed under a Creative Commons Attribution 4.0 International License. Read Full License 


\section{Abstract}

Background: Persistent descending mesocolon (PDM) is a case of colonic replacement and adhesion as a result of abnormal fixation of the gastrointestinal tract. In laparoscopic surgery, it is performed when the patient is in an unconscious state. However, laparoscopic surgery, especially robot-assisted surgery, may necessarily need to devise surgical procedures such as anatomical recognition and adhesion detachment. We herein report three patients with PDM that were treated with arthroscopic surgery at our hospital.

Case presentation: Patient 1: a 73-year-old man with sigmoid colon cancer. Laparoscopic sigmoidectomy was performed, and there were no problems encountered (such as difficulty in recognizing the anatomical location) other than adhesion detachment. Robot-assisted surgery was performed to the other two patients. Patient 2: a 62-year-old man with lower rectal cancer and underwent an intersphincteric resection. Patient 3: a 76-year-old man with lower rectal cancer and underwent Hartmann's operation. The surgery duration of these patients took longer, the same with robot-assisted surgeries without PDM.

Conclusions: Robot-assisted surgeries have a large magnifying effect on delicately removing adhesions, which is a characteristic of PDM. Also, some parts are difficult to grasp as a whole, and it takes time to recognize adhesions anatomically and to grasp the whole image. Here, we report our experience with laparoscopic surgery and robot-assisted surgery for left-sided colorectal cancer with PDM.

\section{Background}

Persistent descending mesocolon (PDM) is a fixation abnormality in which the sigmoid colon displaces to the right and adheres to the small mesentery and right pelvis. ${ }^{1}$ During laparotomy, simple adhesions have been identified and dealt with. However, laparoscopic surgery is a difficult operation, especially in recognizing the anatomy; thus, it requires attention. Here, we report laparoscopic surgery and robotassisted surgery for left colorectal cancer with PDM.

\section{Case Presentation}

\section{Patient 1}

Patient 1 was a 73-year-old man with sigmoid colon cancer. The patient had no history of laparotomy. The patient's body weight, height, and BMI were $53 \mathrm{~kg}, 166 \mathrm{~cm}$, and $19.5 \mathrm{~kg} / \mathrm{m}^{2}$, respectively. Laparoscopic sigmoidectomy was performed. The sigmoid colon extensively adhered to the left abdominal wall to the left pelvic wall, and the small intestinal mesentery at the terminal ileum extensively adhered to the sigmoid colon (Fig. 1a). The descending colon was not fixed and was located slightly to the left of the midline (Fig. 1b). The adhesions were peeled off as much as possible. The medial approach proceeded from the vicinity of promontorium, which is normal anatomy, to the cranial side. Because it was difficult to confirm the blood vessel was running, lymph node dissection was performed near the root of the inferior mesenteric artery (IMA). The blood vessel was ligated and cut off on the distal 
side of the left colic artery (LCA) to maintain blood flow. The operation time was $219 \mathrm{~min}$, and the blood loss was $21 \mathrm{~mL}$.

\section{Patient 2}

Patient 2 was a 62-year-old with lower rectal cancer. The patient had no history of laparotomy. The patient's body weight, height, and BMI were $62 \mathrm{~kg}, 161 \mathrm{~cm}, 22.5 \mathrm{~kg} / \mathrm{m}^{2}$. Robot-assisted intersphincteric resection was performed. Because of an abnormal fixation, the descending colon was located slightly to the left of the midline. No descending colon was present in the left flank, and the peritoneal organ, left gonad, and ureter were visible (Fig. 2a). The sigmoid colon is displaced to the right and adhered to the appendix, ascending colon, and terminal ileum. The adhelysis was laparoscopically performed before the docking to the robotic system (Fig. 2b,c). Although it was difficult to remove the adhesions due to abnormal fixation, the IMA root was identified and then ligated and cut at the same site. The operation time was $525 \mathrm{~min}$, and the blood loss was $80 \mathrm{~mL}$.

\section{Patient 3}

Patient 3 was a 76-year-old man with lower rectal cancer. The patient's body weight, height, and BMI were $52 \mathrm{~kg}, 155 \mathrm{~cm}, 22.0 \mathrm{~kg} / \mathrm{m}^{2}$. The patient had a combination of esophageal cancer and lung cancer. The patient underwent robotic Hartmann's operation with lymph node dissection in the D2 range. It was not fixed because of an abnormal fixation of the descending colon. Moreover, the sigmoid colon was extensively and strongly adherent to the small intestinal mesentery of the midline (Fig. 3a,b). Because of the wide range of adhesions, the robot was docked after the adhesions were detached as much as possible under the laparoscope. After dissection of the adhesions, the anatomy around the IMA was recognized, and then a rectal dissection was performed first. After confirming LCA, sigmoid artery (SA), and superior rectal artery (SRA) from IMA, blood flow was blocked at the planned incision site with vascular clamp forceps; indocyanine green (ICG) fluorescence imaging was used to confirm that there was no blood flow disorder in the remaining planned intestinal tract (Fig. 3c,d). A retroperitoneal incision was made to expose the IMA root from the cranial side of the promontorium, which is normal anatomy. However, because it was difficult to identify the tissue layer in the retroperitoneum, we changed to the lateral approach midway through, identified the gonadal arteries and veins and the ureter from the outside, and performed ablation from the outside. The operation time was $415 \mathrm{~min}$, and the blood loss was $25 \mathrm{~mL}$.

In all patients, we confirmed that the intestinal blood flow was good by using ICG fluorescence imaging before anastomosis.

\section{Discussion And Conclusions}

An abnormal fixation of the mesentery in the fetal period can cause colonic malpositioning but is rare in the left colon. The mesentery is not fixed until the fourth month of pregnancy, but the mesentery of the ascending and descending colons is fixed to the retroperitoneum during the fifth month of pregnancy. ${ }^{1}$ 
PDM is thought to prevent attachment of the midcolon to the parietal peritoneum during this development. ${ }^{1-3}$ Imaging findings are characterized by displacement findings from the left colon to the right side ${ }^{1-3}$ due to immobilization; however, there are reports of right sigmoid colon. ${ }^{4}$ Morgenstern ${ }^{2}$ classified PDM into transverse colon-deficient type, behavioral-variant type, and paracecal type. The cases experienced this time are considered to be classified into left displacement type and paracecal type. In our case, because adhesions are often highly advanced, careful separation operation is necessary to avoid collateral damage during adhesion exfoliation.

Also, the IMA branch may be a characteristic of the radial branches of LCA, SA, and SRA. The LCA forms part of the limbic artery, and the LCA and other limbic arteries may be near each other. ${ }^{5,6}$ Laparotomy had a wide field of view, and the operation was performed while grasping the entire image, so there was no problem. However, laparoscopic surgery has a limited field of view, so anatomical grasping and recognition may be difficult; therefore, caution is required. Also, as mentioned previously, the anomaly is a characteristic of IMA; thus, more caution is required. With the support of robots, the proximity effect and magnification effect are more effective than laparoscopic surgery; however, the observation field of view becomes narrower depending on the site than laparoscopic surgery, making it difficult to grasp the overall image. This can take additional time, causing unintended bleeding and complications such as organ damage. In the experienced case, we tried to recognize blood vessel travel as much as possible. There were also reports that performing radioisotope could assess colonic blood flow and reduce suture insufficiency. ${ }^{7,8}$ ICG fluorescence imaging showed good intestinal blood flow in all cases. It was confirmed that there were no complications due to impaired blood flow, such as an anastomotic leak, intestinal necrosis, or colostomy. Confirmation of blood flow during surgery is very useful for patients where there is a high possibility of abnormal blood vessels, such as this case. In the case where the LCA and the peripheral artery are close to each other, as described previously, it is possible to prevent the peripheral artery from being accidentally cut off by confirming the blood flow running. In the case of arthroscopic surgery for colorectal cancer with PDM, consider the indication of tumor resection, pay close attention to anatomical abnormalities, and select surgical procedures and approaches that do not cause complications. This will be necessary in each case.

\section{Abbreviations}

PDM: Persistent descending mesocolon

IMA: Inferior mesenteric artery

LCA: Left colic artery

SA: Sigmoid artery

SRA: Superior rectal artery

ICG: Indocyanine green 


\section{Declarations}

\section{Ethics declarations}

\section{Ethics approval and consent to participate}

The need for ethic approval is not subject to the institutional review board due to simple case reporting. Written informed consent was obtained from the patients to report and publish individual patient data.

\section{Consent for publication}

Written informed consent was obtained from the patients for publication of this case report and accompanying images.

\section{Availability of data and materials}

All data generated or analyzed during this report are included in this published article. The data can be obtained by corresponding author.

\section{Competing interests}

The authors declare that they have no competing interests.

\section{Funding}

Not applicable.

\section{Authors' contributions}

$\mathrm{KS}, \mathrm{MK}, \mathrm{YO}, \mathrm{KK}$ and AK were mainly involved in the patient treatment/surgery. KS and MK were involved in patient treatment/follow-up. All authors read and approved the final manuscript.

\section{Acknowledgements}

Not applicable.

\section{Author information}

\section{Affiliations}

Department of Coloproctological Surgery, Juntendo University Faculty of Medicine

2-1-1 Hongo, Bunkyo-ku, Tokyo, Japan, 113-8421

Yutaka Kojima, Kazuhiro Sakamoto, Yuichi Tomiki, Masaya Kawai, Yu Okazawa, Ryoichi Tsukamoto, Yuki Tsuchiya 


\section{Corresponding author}

Correspondence to Yutaka Kojima

\section{References}

1. Popky GL, Lapayowker MS. Persisitent descending mesocolon. Radiology. 1966;86:327-31.

2. Morgenstern L. Persisitent descending mesocolon. Surg Gynecol Obstet. 1960;10:197-202.

3. Balthazar EJ. Congenital Positional Anomalies of the Colon: Radiographic Diagnosis and Clinical Implications. I. Abnormalities of Rotation. Gastrointest Radiol. 1977;2:49-56.

4. wondrak E. Right-side fixation of the sigmoid colon. Rozhi Chir. 1957;36:158-60.

5. Wang L, Kondo H, Hirano Y, Ishii T, Hara K, Obara N, et al: Persistent descending mesocolon as a key risk factor in laparoscopic colorectal cancer surgery. In vivo 2020; 34: 807 - 13.

6. Hiyoshi Y, Miyamoto Y, Eto K, Nagai Y, Iwatsuki M, Iwagami S, et al. Laparoscopic surgery for colorectal cancer with perwsistent descending mesocolon. World J Surg Oncol. 2019;17:190.

7. Wada T, Kawada K, Takahashi R, Yoshimoto M, Hida K, Hasegawa S, et al. ICG fluorescence imaging for quantitative evaluation of colonic perfusion in laparoscopic colorectal surgery. Surg Endosc. 2017;31:4184-93.

8. Watanabe J, Ishida A, Suwa Y, Suwa H, Ota M, Kunisaki C, et al. Indocyanine green fluorescence imaging to reduce the risk of anastomotic leakage in laparoscopic low anterior resection for rectal cancer: apropensity score-matched cohort study. Surg Endosc. 2020;34:202-8.

\section{Figures}

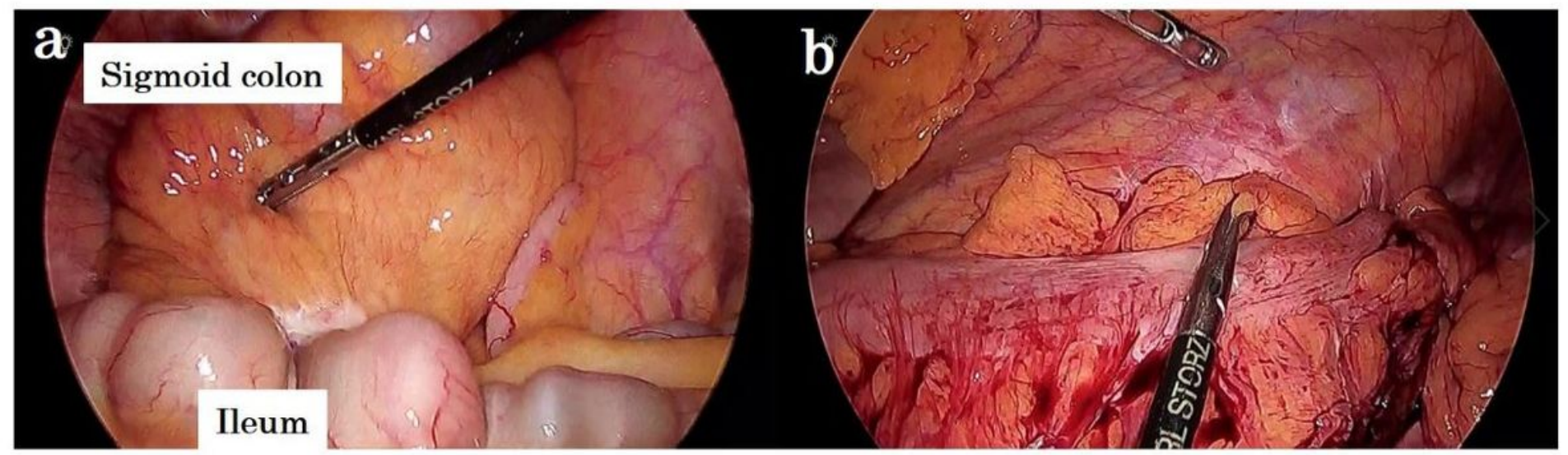

Figure 1

(a) The ileum extensively adhered to the sigmoid mesocolon. (b) The findings after adhelysis showed that the descending colon was not fixed to the left flank and was located in the midline. 


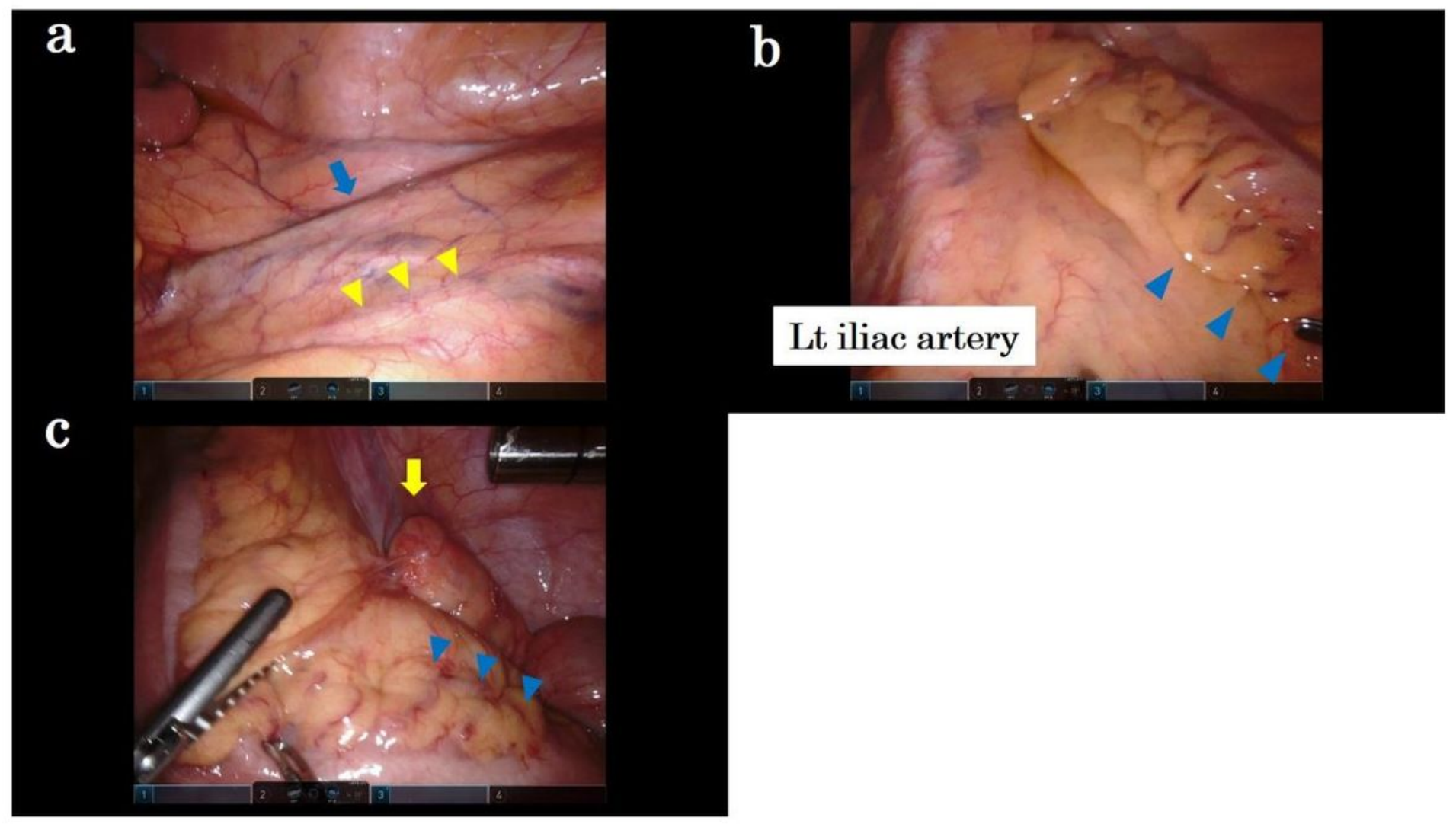

\section{Figure 2}

(a) No descending colon is found in the left lower abdomen, and ureters (arrowhead) and gonadal vessels (arrow) can be confirmed. (b) The sigmoid colon (arrowhead) was displaced from the midline to the left. (c) The sigmoid colon (arrowhead) was strongly adherent to the appendix (arrow) and ascending colon. 


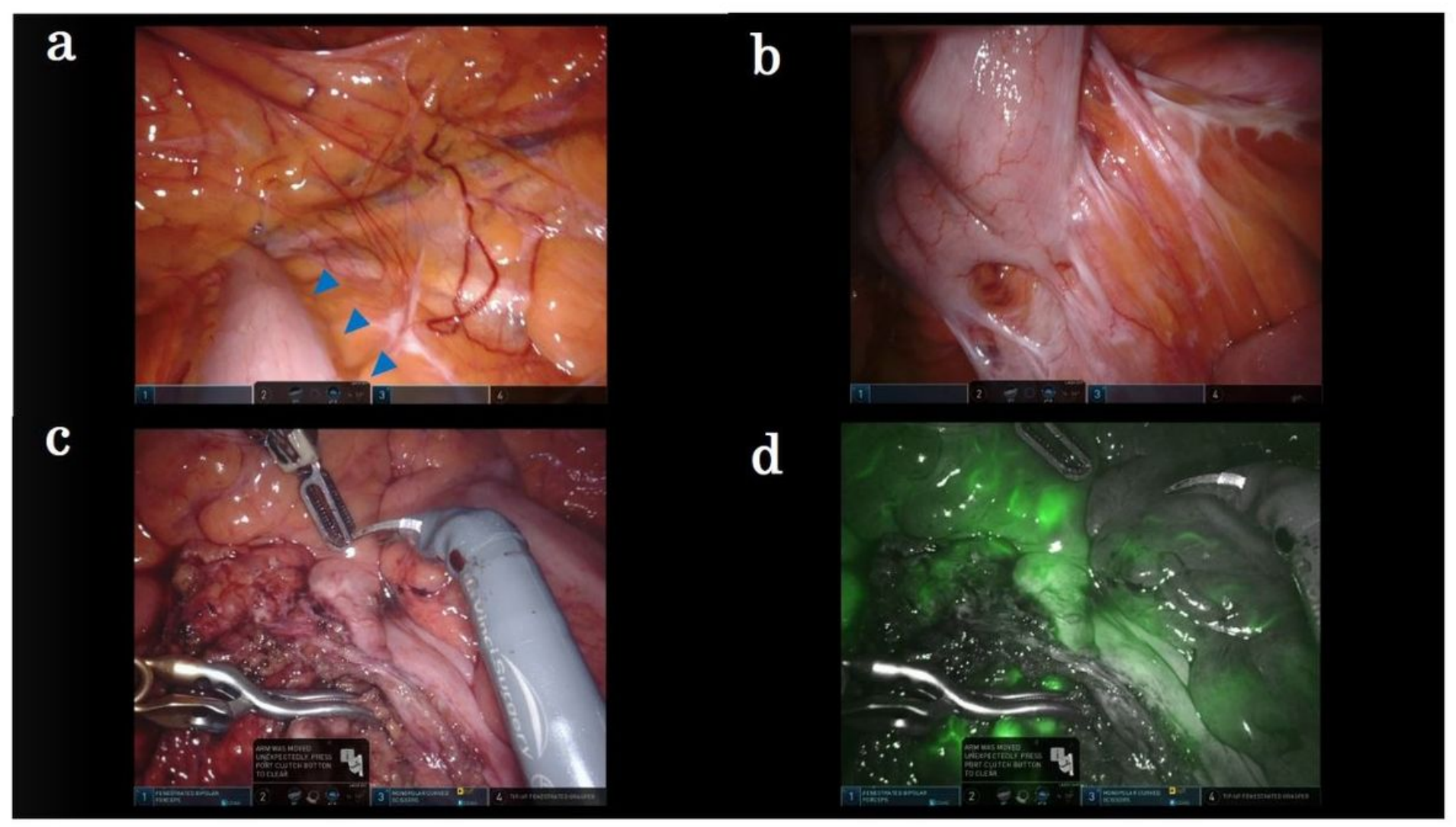

\section{Figure 3}

$(a, b)$ The descending colon (arrowhead) was not fixed and was displaced midway. The descending colon and sigmoid colon were strongly adherent to the small mesentery. (c, d) A blood vessel clip was attached to the planned site of blood vessel dissection, and it was confirmed by ICG fluorescence imaging that there was no abnormality in intestinal blood flow. 